INLO-PUB-02/01

FSU TPI-04/01

\title{
Classical Gauge Vacua as Knots
}

\author{
Pierre van Baal \\ Instituut-Lorentz for Theoretical Physics, University of Leiden, \\ P.O.Box 9506, NL-2300 RA Leiden, The Netherlands \\ Andreas Wipf \\ Friedrich-Schiller-Universität Jena, Theoretisch-Physikalisches Institut, \\ Max-Wien-Platz 1, D-07743 Jena, Germany
}

\begin{abstract}
The four dimensional $O(3)$ non-linear sigma model introduced by Faddeev and Niemi, with a Skyrme-like higher order term to stabilise static knot solutions classified by the Hopf invariant, can be rewritten in terms of the complex two-component $C P_{1}$ variables. A further rewriting of these variables in terms of $S U(2)$ curvature free gauge fields is performed. This leads us to interpret $S U(2)$ pure gauge vacuum configurations, in a particular maximal abelian gauge, in terms of knots with the Hopf invariant equal to the winding number of the gauge configuration.
\end{abstract}

\section{Introduction}

In this Letter we address some simple results that involve rewriting the Faddeev-Niemi model [1]. This model has stable static solutions that represent knots. Much work has been invested in interpreting this model as an effective low-energy representation of $S U(2)$ gauge theory [2, 3, 4] and investigating the quality of this approximation by inverse Monte Carlo techniques [0]. This interpretation in part is motivated by 't Hooft's notion of abelian projection [6].

The model is defined in terms of a space-time dependent vector $\vec{n}(x)$ of fixed (here chosen unit) length. To allow for non-trivial static solutions a Skyrme-like higherorder term is added [7], through the introduction of a composite gauge field strength $F_{\mu \nu}(x)=\frac{1}{2} \vec{n}(x) \cdot\left(\partial_{\mu} \vec{n}(x) \wedge \partial_{\nu} \vec{n}(x)\right)$. Note that with $\vec{n}(x)$ a unit three-vector, $\partial_{\mu} \vec{n}(x)$ 
is perpendicular to $\vec{n}(x)$, and $\partial_{\mu} \vec{n}(x) \wedge \partial_{\nu} \vec{n}(x)$ is proportional to $\vec{n}(x)$. The factor of proportionality is precisely $2 F_{\mu \nu}(x)$. Thus, one also has $F_{\mu \nu}^{2}(x)=\frac{1}{4}\left(\partial_{\mu} \vec{n}(x) \wedge \partial_{\nu} \vec{n}(x)\right)^{2}$. The action is given by

$$
S=\int d^{4} x\left(m^{2} \partial_{\mu} \vec{n}(x) \cdot \partial^{\mu} \vec{n}(x)-\frac{1}{2 e^{2}} F_{\mu \nu}(x) F^{\mu \nu}(x)\right) .
$$

By rescaling $x$ with $(e m)^{-1}, e^{2} S$ becomes independent of both $e$ and $m$. With this understood, we will now put $e=m=1$. Finite energy requires $\vec{n}(\vec{x})$ to approach a constant vector at spatial infinity. In this way static configurations are classified by the topological maps from $S^{3}$ into $S^{2}$, characterised by the Hopf invariant. The two-form $F(\vec{x})=\vec{n}(\vec{x}) \cdot(d \vec{n}(\vec{x}) \wedge d \vec{n}(\vec{x}))$ implicitly defines an abelian gauge field oneform $A(\vec{x})$ through $F(\vec{x})=d A(\vec{x})$, in terms of which the Hopf invariant is given by $Q=\frac{1}{4 \pi^{2}} \int A(\vec{x}) \wedge F(\vec{x})$. Remarkably, the energy is bounded by a fractional power of this Hopf invariant [8, 9].

$$
E=\int d^{3} x\left(\left(\partial_{i} \vec{n}(x)\right)^{2}+\frac{1}{2} F_{i j}^{2}(x)\right) \geq c|Q|^{3 / 4}
$$

with $c=16 \pi^{2} 3^{3 / 8} \sim 238$. This gives a rough bound, which can be improved on 10 (by roughly a factor 2). Extensive numerical studies [11, 12] have gone up to $Q=8$, with energies indeed following the fractional power of $Q$.

\section{The $C P_{1}$ formulation}

We first discuss the reformulation in terms of $C P_{1}$ fields, as well-known from two dimensions [13, 14. The main advantage is that the abelian gauge field involved in defining the Hopf invariant, no longer needs to be defined implicitly. To be specific, one introduces a complex two-component field $\Psi(x)$. The two degrees of freedom associated to the $n$ field are obtained by identifying any two $\Psi$ 's which differ by an overall nonvanishing complex scale factor. This is achieved by constraining $\Psi$ to have unit length, and introducing local abelian gauge invariance, obvious from the following relation to the $n$ field:

$$
n^{a}(x)=\Psi^{\dagger}(x) \tau^{a} \Psi(x),
$$

where $\tau^{a}$ are the Pauli-matrices. The abelian gauge invariance of the $C P_{1}$ model leads to a composite gauge field

$$
A_{\mu}(x)=-i \Psi^{\dagger}(x) \partial_{\mu} \Psi(x)
$$

and one verifies by direct computation that indeed $F(x)=d A(x)$. Useful identities for these computations are the completeness relation $\delta_{i j} \delta_{k l}+\tau_{i j}^{a} \tau_{k l}^{a}=2 \delta_{i l} \delta_{j k}$ and $i \varepsilon_{a b c} \tau_{i j}^{b} \tau_{k l}^{c}=$ 
$\tau_{k j}^{a} \delta_{i l}-\tau_{i l}^{a} \delta_{j k}$. For the action, Eq. (1), we find the following result

$$
S=\int d^{4} x\left(4\left(D_{\mu} \Psi\right)^{\dagger}(x) D^{\mu} \Psi(x)-\frac{1}{2} F_{\mu \nu}(x) F^{\mu \nu}(x)\right)
$$

where $D_{\mu}=\partial_{\mu}-i A_{\mu}(x)$ is the covariant derivative. Note that $\Psi^{\dagger}(x) D_{\mu} \Psi(x)=0$ and that the energy density can be written as a square, $E=\int d^{3} x\left|\left(2 D_{i}+B_{i}(\vec{x})\right) \Psi(\vec{x})\right|^{2}$.

\section{The $S U(2) / U(1)$ formulation}

The next reformulation makes use of the fact that any two-component complex vector of unit length is in one to one relation to an $S U(2)$ group element. Alternatively we can write $\Psi(x)=g(x) \Psi_{0}$. For convenience we choose $\Psi_{0}^{\dagger}=(1,0)$, such that

$$
n_{a}(x)=\frac{1}{2} \operatorname{tr}\left(\tau_{3} g^{\dagger}(x) \tau_{a} g(x)\right)
$$

As we will see, the winding number of $g(\vec{x})$ as a map from $R^{3}$ to $S U(2)$ is precisely the Hopf invariant. This observation is in itself not new [1]]. But we will push it a little further here.

We introduce currents $J_{\mu}^{a}(x)$ through $J_{\mu}(x)=i \tau_{a} J_{\mu}^{a}(x)=g^{\dagger}(x) \partial_{\mu} g(x)$. A simple calculation shows that

$$
A_{\mu}(x)=J_{\mu}^{3}(x) \quad \text { and } \quad \partial_{\mu} \Psi^{\dagger}(x) \partial^{\mu} \Psi(x)=J_{\mu}^{a}(x) J_{a}^{\mu}(x)
$$

We can interpret the currents just as well as components of an $S U(2)$ gauge connection, which is pure gauge, $G(x)=d J(x)+J(x) \wedge J(x)=0$, with $J(x) \equiv J_{\mu}(x) d x_{\mu}$. For later use we also introduce $J^{a}(x) \equiv J_{\mu}^{a}(x) d x_{\mu}$. In particular in components, we have $G_{\mu \nu}^{3}(x)=\partial_{\mu} J_{\nu}^{3}(x)-\partial_{\nu} J_{\mu}^{3}(x)-2\left(J_{\mu}^{1}(x) J_{\nu}^{2}(x)-J_{\nu}^{1}(x) J_{\mu}^{2}(x)\right)=0$. It leads to the useful identity

$$
F(x)=d J^{3}(x)=2 J^{1}(x) \wedge J^{2}(x) \quad \text { or } \quad F_{\mu \nu}(x)=2\left(J_{\mu}^{1}(x) J_{\nu}^{2}(x)-J_{\nu}^{1}(x) J_{\mu}^{2}(x)\right) .
$$

With the help of this relation it is now also easy to show that the Hopf invariant is exactly equal to the winding number of the gauge function $g(\vec{x})$,

$$
\frac{1}{4 \pi^{2}} A(\vec{x}) \wedge F(\vec{x})=\frac{1}{2 \pi^{2}} J^{3}(\vec{x}) \wedge J^{1}(\vec{x}) \wedge J^{2}(\vec{x})=\frac{1}{24 \pi^{2}} \operatorname{tr}\left(g^{\dagger}(\vec{x}) d g(\vec{x})\right)^{3},
$$

which can of course also be related to the non-abelian Chern-Simons form,

$$
\frac{1}{4 \pi^{2}} A(\vec{x}) \wedge F(\vec{x})=-\frac{1}{8 \pi^{2}} \operatorname{tr}\left(J(\vec{x}) \wedge d J(\vec{x})+\frac{2}{3} J(\vec{x}) \wedge J(\vec{x}) \wedge J(\vec{x})\right) .
$$


A similar relation between the Hopf invariant and a non-Abelian Chern-Simons form was discussed in Ref. [3]. But we wish to argue here that the static solitons of the Faddeev-Niemi model actually represent classical Yang-Mills vacua in a non-linear maximally abelian gauge. It is this result that we believe to be new.

First we note that,

$$
\left(D_{\mu} \Psi\right)^{\dagger}(x) D^{\mu} \Psi(x)=\partial_{\mu} \Psi^{\dagger}(x) \partial^{\mu} \Psi(x)-A_{\mu}(x) A^{\mu}(x)=J_{\mu}^{1}(x) J_{1}^{\mu}(x)+J_{\mu}^{2}(x) J_{2}^{\mu}(x),
$$

which makes the $S U(2) / U(1)$ nature of the action explicit, since both terms in Eq. (5) can be written in terms of just $J_{\mu}^{1}(x)$ and $J_{\mu}^{2}(x)$. So the energy of a static configuration is given in terms of the "charged" components of the non-abelian gauge field only

$$
E=\int d^{3} x\left(4\left(J_{i}^{1}(\vec{x}) J_{i}^{1}(\vec{x})+J_{i}^{2}(\vec{x}) J_{i}^{2}(\vec{x})\right)+2\left(J_{i}^{1}(\vec{x}) J_{j}^{2}(\vec{x})-J_{j}^{1}(\vec{x}) J_{i}^{2}(\vec{x})\right)^{2}\right)
$$

The first term agrees exactly with the functional that defines the maximal abelian gauge, by minimising along the gauge orbit, leaving the abelian subgroup generated by $\tau_{3}$ unfixed [6, [15]. This remains true for the full energy functional, which can thus just as well be interpreted as the gauge fixing functional for a non-linear maximal abelian gauge. As the three parametrisations are mathematically equivalent, we are entitled to interpret the minima of the energy functional in the sector with a given value of $Q$ as gauge fixed pure gauge (i.e. curvature free, or flat) connections in a sector with gauge field winding number $Q$. Therefore, there is a gauge fixing in terms of which the gauge vacua with different winding number can be characterised by inequivalent knots.

\section{Conclusions}

In the light of the attempts to relate the Faddeev-Niemi model to full non-abelian gauge theory, our result is a rather sobering one, even though it also involves an abelian projection. Within the context of our interpretation, there seems not much need to address the quantum fluctuations. It should, however, be noted that at the quantum level the three models are not equivalent, as the path integral measure depends on the chosen representation. It is the measure that seems to cause some of the problems in relating the Faddeev-Niemi model to the full $S U(2)$ gauge theory.

We hope this Letter provides inspiration for new ways of viewing the topological non-trivial nature of non-abelian gauge theories. The relation of pure gauge theory vacua to knots is also suggestive from the point of view of Chern-Simons theory and topological field theory. Instantons become knot changing operations, as also suggested in Ref. [3], and one may even hope the present results can have some mathematical ramifications [16, 17]. We will leave this to future studies. 


\section{Acknowledgements}

P.v.B thanks Leo Stodolsky, Valya Zakharov and Dieter Maison for discussions and hospitality at the MPI in Munich, where this work was first presented. A.W. thanks the Lorentz-Institute in Leiden, where much of the work has been done, for excellent working conditions and Falk Bruckmann for many useful discussions.

\section{References}

[1] L. Faddeev and A. J. Niemi, Nature 387 (1997) 58 hep-th/9610193.

[2] L. Faddeev and A. J. Niemi, Phys. Rev. Lett. 82 (1999) 1624 [hep-th/9807069].

[3] E. Langmann and A. J. Niemi, Phys. Lett. B 463 (1999) 252 [hep-th/9905147.

[4] Y. M. Cho, H. Lee and D. G. Pak, "Effective theory of QCD," hep-th/9905215.

[5] L. Dittmann, T. Heinzl and A. Wipf, to appear.

[6] G. 't Hooft, Nucl. Phys. B 190 (1981) 455.

[7] L. D. Faddeev, "Quantization of Solitons," Print-75-0570 (IAS, Princeton).

[8] A. F. Vakulenko and L. V. Kapitansky, Sov. Phys. Dokl. 24 (1979) 433.

[9] A. Kundu and Y. P. Rybakov, J. Phys. A 15 (1982) 269.

[10] R. S. Ward, Nonlinearity 12 (1999) 1 [hep-th/9811176].

[11] R. A. Battye and P. M. Sutcliffe, Phys. Rev. Lett. 81 (1998) 4798 hep-th/9808129;

Proc. Roy. Soc. Lond. A 455 (1999) 4305 [hep-th/9811077].

[12] J. Hietarinta and P. Salo, Phys. Rev. D 62 (2000) 081701.

[13] H. Eichenherr, Nucl. Phys. B 146 (1978) 215 [Erratum-ibid. B 155 (1978) 544].

[14] A. D'Adda, M. Lüscher and P. Di Vecchia, Nucl. Phys. B 146 (1978) 63.

[15] A. S. Kronfeld, M. L. Laursen, G. Schierholz and U. J. Wiese, Phys. Lett. B 198 (1987) 516.

[16] E. Witten, Commun. Math. Phys. 121 (1989) 351.

[17] P. van Baal, Acta Phys. Polon. B 21 (1990) 73. 